\title{
Transferência de conhecimento para o cuidado de enfermagem a pessoa com doença de parkinson
}

\section{$F$ knowledge transfer for nursing care for people with parkinson's disease}

\author{
Simony Fabíola Lopes Nunes' • Angela Maria Alvarez ${ }^{2} \bullet$ Rafaela Vivian Valcarenghi $^{3}$ \\ Maria Fernanda Baeta Neves Alonso da Costa $^{4} \bullet$ Rafaela Baptista $^{5}$
}

\begin{abstract}
RESUMO
Objetivo: identificar na literatura a adoção de transferência e intercâmbio de conhecimento para o cuidado de enfermagem a pessoa com doença de Parkinson. Método: Revisão integrativa realizada nas bases de dados: Public Medline, Cumulative Index to Nursing \& Allied Health Literature, Web of Science, SciVerse Scopus, Base de Dados de Enfermagem, Literatura Latino-Americano e do Caribe em Ciências da Saúde, e Scientific Electronic Library Online, sem recorte temporal pré-estabelecido, em português, inglês e espanhol. Utilizou-se dos termos "Doença de Parkinson", “Enfermagem”, "Prática Clínica Baseada na Evidência", que foram combinados com os operadores booleanos "AND", "OR" e "NOT". Resultados: 16 estudos sobre melhores práticas de enfermagem desenvolvidas para as pessoas que vivem com doença de Parkinson e ou seus familiares foram elegíveis para inclusão neste estudo. Esta revisão identificou que a adoção de práticas baseada em evidências por enfermeiros no cuidado a pessoa com doença de Parkinson envolve elaboração de diretrizes e protocolos de cuidado, mútua ação com a equipe multiprofissional e engajamento do paciente e seus familiares no processo de viver com a doença. Conclusão: As estratégias de enfermagem demonstraram-se eficazes para o cuidado das pessoas com doença de Parkinson e, possuem potencial para serem implementadas na realidade brasileira.

Descritores: Doença de Parkinson; Enfermagem; Prática clínica baseada na evidência; Pesquisa médica translacional; Enfermagem Baseada em Evidências.
\end{abstract}

\begin{abstract}
Objective:To know the production of knowledge in the adoption of evidence-based practices by nurses in the care of the person with Parkinson's disease. Methods: Integrative review using a search strategy in databases: Public Medline, Cumulative Index to Nursing \& Allied Health Literature, Web of Science, SciVerse Scopus, Nursing Database, Latin American and Caribbean Literature in Health Sciences, and Scientific Electronic Library Online, using the terms "Parkinson's Disease","Nursing", "Clinical Practice Evidence-Based". Results: 16 studies on best nursing practices developed for people living with Parkinson's disease and or their families were eligible for inclusion in this study. This review identified that the adoption of evidence-based practices by nurses in the care of people with Parkinson's disease involves the elaboration of care guidelines and protocols, mutual action with the multidisciplinary team and the engagement of the patient and his family in the process of living with the disease. Conclusion: Nursing strategies have proven to be effective for the care of people with Parkinson's disease and have the potential to be implemented in the Brazilian reality.

Keywords: Parkinson's disease; Nursing; Evidence-based clinical practice; Translational medical research; Evidence-Based Nursing.
\end{abstract}

I Enfermeira. Mestre em Enfermagem pela Universidade Federal de Santa Catarina (UFSC). Professora do curso de graduação em enfermagem da Universidade Federal do Maranhão (UFMA). Bolsista FAPEMA. Doutoranda em Enfermagem pela Universidade Federal de Santa Catarina (UFSC).Florianópolis, SC, Brasil.E-mail: sflnunes@ hotmail.com ORCID: http://orcid.org/0000-0003-46/3-8542

2 Enfermeira. Doutora em Filosofia da Enfermagem pela Universidade Federal de Santa Catarina (UFSC). Professora Titular da Universidade Federal de Santa Catarina. Líder do laboratório de Pesquisas e Tecnologias em Enfermagem, Cuidado em Saúde a Pessoas Idosas - GESPI/PEN/UFSC. Florianópolis, SC, Brasil. E-mail: angela. alvarez@ufsc.br ORCID: http://orcid.org/0000-0002-2622-3494

3 Enfermeira. Doutora em Enfermagem pela Universidade Federal de Santa Catarina (UFSC). Professora do curso de graduação em enfermagem da Faculdade Santa Catarina. Florianópolis, SC, Brasil. E-mail: rafaelavalcarenghi@yahoo.com.br ORCID: http://orcid.org/0000-0002-7083-3329

4 Enfermeira. Doutora em Ciências da Saúde pela Escola de Enfermagem da Universidade de São Paulo (EEUSP). Professora Adjunto II do Departamento de Enfermagem - Área de Saúde Pública da Universidade Federal de Santa Catarina (UFSC) Coordenadora do Núcleo de Estudos da Terceira Idade (NETI), da Pró-Reitoria de Extensão, Universidade Federal de Santa Catarina. Florianópolis, SC, Brasil. E-mail: fernanda.baeta@ufsc.br ORCID: http://orcid.org/0000-0002-2763-8050

5 Enfermeira. Mestre em Enfermagem pela Universidade Federal de Santa Catarina (UFSC). Chefe da Unidade cirurgia/RPA e CME, junto ao setor de apoio terapêutico, da divisão de apoio diagnóstico e terapêutico, da gerência de atenção à saúde do Hospital Universitário da Universidade Federal de Santa Catarina (HU-UIFSC). Florianópolis, SC, Brasil. E-mail: rafaela_tenbap@yahoo.com.br ORCID: http://orcid.org/0000-000 I-5094-1886 


\section{INTRODUÇÃO}

A doença de Parkinson (DP) é a segunda doença crônica neurodegenerativa mais frequente em todo o mundo. É uma condição progressiva que provoca vários sintomas motores e não motores com efeitos na mudança de vida das pessoas que desenvolvem esta doença, bem como para seus familiares e cuidadores ${ }^{(1)}$.

O cuidado de pessoas com DP pode ser complexo devido à apresentação clínica e sintomatologia distinta, associada ao fato de que muitas vezes são coordenadas por diversos profissionais de saúde, muitos dos quais não são especialistas em DP ou demais desordens motoras ${ }^{(2)}$. As complexidades dos sintomas motores e não-motores e a natureza crônica progressiva da DP evidenciam a necessidade de enfermeiros conhecedores da doença, capazes de resolver as lacunas na prestação de serviços de saúde primários ${ }^{(3)}$.

Apesar de enfermeiros serem sempre presentes no cuidado a pessoa com DP, somente em 2017 foi publicado uma diretriz na Holanda para enfermeiros especialistas em DP. A criação da diretriz foi necessária porque até aquele ano não havia uma diretiva para os enfermeiros holandeses, e por este motivo as intervenções baseavamse exclusivamente na prática clínica diária. Diretrizes como esta fornecem evidências do desenvolvimento de habilidades e competência necessárias para enfermeiros que trabalham com DP, com recomendações baseadas em boas práticas clínicas nas seguintes necessidades comprometidas pela doença: autocuidado, funcionamento mental, mobilidade, nutrição, sexualidade, trabalho, sono, cuidados paliativos e cuidados complementares (integrativos) $^{(4)}$.

A integração das melhores evidências de enfermagem na prestação de cuidados clínicos é essencial para otimizar a qualidade dos cuidados e os resultados dos pacientes, tornando o serviço de saúde mais eficaz e sustentável $^{(5)}$. É também imperativo que enfermeiros especialistas trabalhem com pesquisadores para otimizar a base de evidências para modelos avançados de atendimento que abordam as necessidades do cliente em toda a trajetória da DP, desde o momento do diagnóstico até os cuidados paliativos e de fim de vida ${ }^{(6)}$.

Uma abordagem integrada de translação de conhecimento para pesquisa é a forma de responder a esse chamado à medida que o conhecimento gerado é agregado para aumentar o conhecimento acumulado em pesquisa relevante aos enfermeiros da prática ${ }^{(7)}$.A translação integrada de conhecimento visa responder uma necessidade identificada através da colaboração entre pesquisadores, profissionais, formuladores de políticas e a comunidade para criar e implementar conhecimento, objetivando meIhorar a eficiência no sistema de saúde ${ }^{(8)}$.

Enfermeiros podem fazer diferença significativa na vida das pessoas afetadas pela $\mathrm{DP}^{(9)}$ quando implementa componentes necessários para aperfeiçoar a qualidade do atendimento, seja em situações agudas, em ambientes comunitários ou domiciliários. Assim, o objetivo desta revisão integrativa é apresentar a literatura sobre a adoção de transferência e intercâmbio de conhecimento para o cuidado de enfermagem a pessoa com doença de Parkinson.

\section{MÉTODO}

Estudo descritivo, bibliográfico, do tipo revisão integrativa de literatura. Para seguir o rigor metodológico de pesquisa do tipo revisão integrativa, adotaram-se as seguintes estratégias propostas pelas enfermeiras Whittemore e Knafl ${ }^{(10)}$ : identificação do problema e formulação da questão norteadora; estágio de busca de literatura; coleta de dados; avaliação; análise e interpretação dos dados; apresentação dos resultados.

A questão que norteou a pesquisa foi: I. Quais as evidências cientificas sobre a adoção de transferência e intercâmbio de conhecimento para o cuidado de enfermagem a pessoa com doença de Parkinson?

O processo de busca e seleção dos artigos, foi realizada nas seguintes bases de dados e coleções de bibliotecas virtuais: Public Medline (PubMed), Cumulative Index to Nursing \& Allied Health Literature (CINAHL),Web of Science (WoS), SciVerse Scopus (SCOPUS), Base de Dados de Enfermagem (BDENF), Literatura Latino-Americano e do Caribe em Ciências da Saúde (LILACS), e Scientific Electronic Library Online (SCIELO). $\mathrm{O}$ acesso às bases de dados ocorreu da seguinte maneira: para acessar PubMed/Medline utilizou-se o portal PubMed. Para acessar Web of Science, CINAHL e SCOPUS, utilizou-se o Portal de Periódicos da Coordenação de Aperfeiçoamento de Pessoal de Nível Superior (CAPES); para a LILACS e BDENF o acesso foi via Biblioteca Virtual de Saúde (BVS), para a SCIELO foi o site da própria base de dados. Neste estudo não foram utilizados métodos adicionais à pesquisa de banco de dados para identificar outros estudos relevantes (pesquisas de citações, contato com especialistas, verificação de referências, pesquisa manual, entre outros).

Realizou-se a pesquisa através da utilização dos termos em inglês, espanhol e português conforme os Descritores em Ciências da Saúde (DeCS) e seus correspondentes no Medical Subject Headings (MeSH): Nursing; Parkinson Disease; Evidence-Based Practice. Ressalta-se que a estratégia de busca foi adaptada às bases de dados pesquisadas, seguindo seus critérios de pesquisa que foram combinados com os operadores booleanos "AND", "OR" e "NOT".

A coleta de dados ocorreu nos meses de fevereiro a abril de 2019, e os critérios de inclusão foram: todos os anos de publicação, estudos com resumos na íntegra disponíveis on-line, nos idiomas: português, espanhol ou 
inglês e que focalizassem aspectos relacionados a intervenções estruturadas da prática clínica de enfermagem as pessoas com DP e seus familiares e/ou cuidadores. Excluíram-se publicações classificadas como editoriais, comentários, resenhas de livros, relatos de casos e/ou protocolos de pesquisa de revisão sistemática; estudos que não coletaram, analisaram ou relataram dados primários ou secundários; e estudos que não investigam algum aspecto da prática de enfermagem a pessoa com DP.

A pesquisa gerou $6 \mathrm{I}$ citações. Depois de remover duplicatas através do gerenciador de referências Mendeley Web Importer e aplicar os critérios de elegibilidade, foram incluídos na amostra 16 artigos de periódicos. $O$ processo de busca e recuperação desta revisão está ilustrado na figura $I$.

Para extração de dados e obtenção das informações que respondiam a questão de pesquisa, utilizou-se como instrumento um quadro para organizar e analisar os artigos pré-selecionados, contendo: autoria, ano de publicação e país da pesquisa, participantes, tipo de estudo e síntese principais resultados.

Para análise dos dados ${ }^{(10)}$, o texto completo de todos os estudos foi revisado, e os dados extraídos foram or- ganizados de acordo com temas e relacionados usando comparação constante, para que fossem categorizados e agrupados por semelhança.

Sendo assim, foi realizada a redução dos dados, classificando inicialmente subgrupos por conceitos recorrentes, usuário do conhecimento envolvido na pesquisa e intervenção/comportamento proposto em cada estudo. Na segunda fase da análise dos dados foi realizado a exibição de dados, que envolveu a conversão dos dados extraídos de fontes individuais e relações entre os conceitos, e por fim, descreveu-se as relações entre os conceitos selecionados que gerou a identificação de padrões e temas relacionados ao cuidado de enfermagem a pessoa com DP.

\section{RESULTADOS}

Dos 16 estudos incluídos, todos estavam no idioma inglês. Quanto ao país de origem dos pesquisadores principais seis eram dos Estados Unidos, cinco da Holanda, e um estudo da Austrália, Dinamarca, Reino Unido, Escócia e Canadá, respectivamente. Destaque para Holanda, onde todos os cinco estudos identificados eram de autores que faziam parte do Departamento de Neurologia do

Artigos identificados nas bases de dados MEDLINE/PUBMED ( $\mathrm{n}=49$ ), SCOPUS $(n=4)$, WEB OF SCIENCE $(n=2)$, CINAHL $(n=4)$, LILACS e BDENF $(n=2)$, SCIELO (0) $(\mathrm{n}=61)$

\begin{tabular}{|r|}
$\begin{array}{r}\text { Artigos selecionados para avaliação de } \\
\text { títulos }(\mathrm{n}=57)\end{array}$ \\
\hline $\begin{array}{c}\text { Artigos selecionados para leitura do resumo } \\
(\mathrm{n}=18)\end{array}$ \\
para elegibilidade e selecionados para \\
análise (n=16)
\end{tabular}

Artigos eliminados por estarem duplicados $(n=4)$
Artigos excluídos após leitura dos títulos $(\mathrm{n}=39)$
Artigos excluídos após critérios de elegibilidade $(\mathrm{n}=2)$

1. Não se tratava de intervenção exclusivamente para pessoas com DP;

2. Protocolo de pesquisa de revisão sistemática

FIGURA 1 - Fluxograma de busca na literatura e processo de seleção dos artigos. Florianópolis, Santa Catarina, Brasil. 2019.

Fonte: Resultados da pesquisa (2019). Elaboração dos autores. 
Centro de Prática Baseada em Evidências de Nijmegen. Quanto ao ano de publicação, os artigos datam de 2010 a 2019 e, em relação ao tipo de estudo utilizado, seis utilizaram método mistos, quatro estudos utilizaram métodos quantitativos (sendo ensaios clínicos randomizados), quatro estudos abordagem qualitativa e dois foram revisões de literatura. $O$ quadro I lista a síntese dos estudos incluídos na revisão e suas principais características.

Diante do tipo de adoção de transferência e intercâmbio de conhecimento pesquisado para o cuidado de enfermagem a pessoa com DP, dividiram-se quatro categorias gerais: Programas educacionais na instrumentalização dos enfermeiros para o cuidado das pessoas com DP, Cuidado de enfermagem a pessoa com DP na abordagem interdisciplinar, Protocolos clínicos e diretrizes de enfermagem baseada em evidências para o cuidado a pessoa com DP e Enfermeiros colaboradores na tomada de decisão individual e compartilhada.

\section{DISCUSSÃO}

\section{Programas educacionais na instrumentalização dos enfermeiros para o cuidado das pessoas com DP}

Autores referem que os enfermeiros estão intimamente envolvidos em vários aspectos do atendimento a pessoa com DP, incluindo aconselhamento, enfrentamento da doença e preocupações sociais advindas após o diagnóstico, e para tanto, precisam estar instrumentalizados para gerenciar o cuidado de forma eficaz e atualizada ${ }^{(2)}$.

Intervenções educativas podem ser úteis, por incluir sessões educacionais estruturadas ou programas de educação continuada para instrumentalizar os enfermeiros na prestação de cuidados dentro de um quadro centrado no indivíduo e na família. Nesta revisão, dois estudos incluídos se basearam em programas educacionais continuados, ${ }^{(15,18)}$ e um estudo em estratégias de disseminação passiva para fornecer informações aos enfermeiros e outros profissionais da saúde ${ }^{(19)}$.

Autores $^{(19)}$ desenvolveram um curso para enfermeiros especialistas em DP, baseado em evidências sobre gerenciamento do sono com a finalidade de transferir habilidades em educação em saúde para aumentar o entendimento sobre os distúrbios do sono na doença e melhorar $\mathrm{o}$ atendimento ao paciente. $\mathrm{O}$ curso teve duração de três dias e contemplou assuntos sobre avaliação do sono; prática da higiene do sono; indicação de métodos de relaxamento; uso de controle de estímulo e procedimentos de restrição de sono; e abordagens cognitivas para o gerenciamento da insônia.

Os resultados do programa constataram que os pacientes assistidos pelos enfermeiros que realizaram o curso, apresentaram redução na ansiedade em relação aos problemas do sono, sentindo-se capazes de contro- lar o sono, sugerindo que o modelo de treinamento utilizado é viável e prático; os recursos projetados para uso clínico são funcionais e relevantes; e que a iniciativa em sua totalidade representa um investimento válido e acessível ao bem-estar do paciente ${ }^{(19)}$.

No estudo ${ }^{(18)}$ de intervenção educativa para enfermeiros hospitalares e de cuidados primários através do programa denominado Parkinson's Disease Medication Protocol Program foi considerada eficaz, por aumentar significativamente os níveis de conhecimento dos enfermeiros nos domínios de fisiopatologia, medicação e cuidados de enfermagem.

Autores $^{(15)}$ argumentam que profissionais de saúde precisam de educação em práticas de DP baseadas em evidências para trabalhar efetivamente em equipes e gerenciar adequadamente a doença, e assim mudar a prática clínica. Em estudo multicêntrico, foi desenvolvido um programa de educação interprofissional com um protocolo de ensino replicável, que gerou mudanças positivas na prática de enfermeiros e demais membros da equipe, como: desenvolvimento de clínica especializada em DP; fortalecimento da equipe ou implantação de rede interprofissional; criação de novos programas ou serviços de DP, e adoção de abordagem em equipe para suprir o atendimento fragmentado da DP.

Através destes resultados sobre programas educacionais, verifica-se que enfermeiros capacitados conseguem entender a mudança de paradigma, desenvolvendo habilidades para antecipar, identificar e remover barreiras que incentivam a implementação e manutenção das mudanças na prática.

\section{Cuidado de enfermagem a pessoa com DP na abordagem interdisciplinar}

A evidência da eficácia do cuidado de enfermagem para pessoas com DP permanece limitada e inconclusiva, demonstrando que este, seja mais eficaz quando associado a uma abordagem de equipe interdisciplinar ${ }^{(2)}$. Nesta revisão, três estudos ${ }^{(2,12,20)}$ direcionaram as evidências das intervenções de enfermagem para $\circ$ atendimento multidisciplinar de pacientes com DP.

A natureza multifacetada da DP exige um modelo de atendimento personalizado e orientado para a equipe, no entanto, modelos baseados em evidências que descrevam como os cuidados com a multiespecialidade devem ser organizados são limitados ${ }^{(2,16)}$. O mais próximo encontrado na síntese desta revisão foi um estudo de pesquisadores de New York sobre visitas domiciliares interdisciplinares para indivíduos com DP no estágio avançado ${ }^{(12)}$.

A investigação detalhou o programa de visitas domiciliares interdisciplinares projetados especificamente para indivíduos com DP e transtornos relacionados e seus cuidadores familiares, com base nos princípios de me- 
Ihores práticas no cuidado de idosos. A visita domiciliar tinha periodicidade trimestral, e a equipe era composta por neurologista, assistente social, e enfermeiro que trabalhavam em paralelo com a pessoa com DP e seu cuidador para completar a anamnese, adequação dos medicamentos, avaliação física, avaliação das necessidades psicossociais e da segurança domiciliar, finalizando em um plano de cuidado interdisciplinar ${ }^{(12)}$.

Os autores mostraram ainda que, algumas atividades de enfermagem descritas poderiam ser adaptadas a outros contextos locais que possuem atendimento domiciliar, a destacar: avaliação dos sinais vitais, incluindo a pressão arterial ortostática, devido à sua prevalência e quedas associadas; avaliação da medicação, revendo a compreensão do paciente sobre o manejo da polifarmácia; avaliação de segurança da casa para identificar possíveis riscos de queda e riscos de segurança ${ }^{(12)}$.

Lindop ${ }^{(20)}$ também listou alguns cuidados de enfermagem a pessoa com DP que poderiam ser adaptados a contextos que possuem atendimento domiciliar, como: avaliação do plano medicamentoso, mensuração da pressão sanguínea, peso e postura; inquérito sobre deglutição, sono, função sexual, constipação, fala e histórico de quedas; contato para consulta na clínica-virtual; reunião mensal com a equipe interdisciplinar para elaboração de programa de terapia, metas de reabilitação e planos de tratamento; visita domiciliar; e, encaminhamento para serviço social ou tratamento hospitalar quando necessário.

Para gerenciar as complexas demandas da DP, um estudo controlado randomizado realizado no Centro de Distúrbios do Movimento na Holanda constatou que pessoas com DP acompanhadas no período de oito meses de intervenção multidisciplinar especializada (especialista em distúrbios do movimento, enfermeiro especialista em DP e assistente social) obteve melhoras das medidas de qualidade de vida (avaliadas através da escala Parkinson's Disease Quality of Life Scale - PDQ-39) e nos escores do funcionamento motor (avaliados pela Escala Unificada de Avaliação da Doença de Parkinson/UPDRS III) em comparação com manejo daqueles acompanhados apenas por um neurologista ${ }^{(2)}$.

Os resultados desta categoria revelam que o cuidado de enfermagem a pessoa com DP na abordagem interdisciplinar está consolidado em países desenvolvidos e onde existe a denominação "enfermeiros especialistas na doença de Parkinson”.

\section{Protocolos clínicos e diretrizes de enfermagem baseada em evidências para o cuidado a pessoa com DP}

A aprendizagem que ocorre durante o relato do processo de implementação da prática baseada em evidência ou práticas inovadoras dentro de contextos locais são informações valiosas. Tais experiências servem de aprendizagem para melhorias de processos locais e orientar tomadores de decisão sobre estratégias de implementação bem-sucedida baseadas em evidências para uso em outros contextos de prática ${ }^{(24)}$.

Nesta revisão, quatro estudos identificados tratavam sobre desenvolvimento de recomendações e diretrizes clínicas baseadas em evidências de enfermagem para meIhorar a qualidade de saúde da pessoa com DP. Foram eles: gerenciamento da saúde bucal para pacientes com $D^{(10)}$, recomendações para prática clínica de enfermeiros especialista na $\mathrm{DP}^{(4)}$, consenso para exame e tratamento de quedas em pessoas com DP(17), manejo do paciente com instabilidade da pressão arterial na $\mathrm{DP}^{(23)}$.

Para tratar eficazmente a doença, pesquisadores da Sociedade Holandesa de Enfermeiros Especialistas na Doença de Parkinson e da rede holandesa de assistência médica nacional ParkinsonNet, formularam diretriz para enfermeiros com intervenções fundamentadas exclusivamente na prática clínica diária. A diretriz foi respaldada em uma revisão de literatura e um questionário preenchido por 97 enfermeiros especialistas na DP e 5 I enfermeiros generalistas com conhecimento sobre a doença para identificar barreiras no cuidado de enfermagem em $\mathrm{DP}^{(4)}$.

Os membro da Sociedade Holandesa de Enfermeiros Especialistas na Doença de Parkinson e do ParkinsonNet definiram tarefas primárias para os enfermeiros no atendimento padrão aos pacientes e seus cuidadores, sendo as principais: fornecer informações e educação; apoiar paciente e o cuidador na auto-gestão da doença; apoiar pacientes e cuidadores nos domínios psicossocial e existencial; trabalhar em colaboração multidisciplinar; e, realizar intervenções técnicas específicas de enfermagem ${ }^{(4)}$.

Protocolo de autocuidado pode auxiliar no planejamento de esquemas de atendimento mais eficazes em doenças neurodegenerativas como a DP(23). Neste sentido, reconhecendo que a prevenção de quedas é elemento importante da qualidade do atendimento as pessoas com DP, pesquisadores holandeses produziram um conjunto abrangente de recomendações para enfermeiros e outros profissionais de saúde, visando a redução de quedas para pessoas com a doença ${ }^{(17)}$.

As recomendações de prevenção de quedas foram adaptadas a partir da combinação de fatores genéricos de risco para idosos e fatores de risco específicos para DP, incluindo $3 \mathrm{I}$ fatores de risco ( 16 fatores de risco genéricos e 15 fatores de risco específicos). Os fatores de risco genéricos para quedas na DP (idade, sexo, medicamentos, polifarmácia) foram recomendados para serem gerenciados pelo clínico geral, geriatra, neurologista e enfermeiro especialista em DP. Enquanto que o neurologista, enfermeiro especialista em DP e fisioterapeuta foram considerados os principais profissionais para abordar os 
fatores de risco específicos (gravidade da doença, mobilidade lenta, congelamento da marcha dentre outros da DP) ${ }^{(17)}$.

Pesquisadores canadenses defendem que a translação integrada do conhecimento é um método no qual a inovação é incorporada à prática, devendo envolver a colaboração ativa entre pesquisadores (produtores de conhecimento) e pacientes, formuladores de políticas e/ ou outros tomadores de decisão (também denominados usuários do conhecimento) em vários estágios do processo de pesquisa ${ }^{(25)}$. Ao ser configurada com foco no problema e na lacuna do conhecimento existente, as diretrizes clínicas fornecem base para harmonizar a prestação de cuidados pelo enfermeiro na prática clínica, além de oferecer base para investigações futuras ${ }^{(24)}$.

Nesta pesquisa, somente uma publicação concluiu com recomendações adicionais para pesquisa, política de saúde e prática clínica ${ }^{(23)}$, e apenas o estudo sobre diretrizes para a saúde bucal em indivíduos com DP descreveram a inclusão de diversos usuários do conhecimento (pacientes, cuidadores, enfermeiros e higienistas dentais) na elaboração da diretriz, bem como avaliou a acessibilidade das orientações contidas nas diretrizes ${ }^{(10)}$.

\section{Enfermeiros colaboradores na tomada de decisão individual e compartilhada}

Os tomadores de decisão têm papel relevante para a translação do conhecimento porque auxiliam pesquisadores e formuladores de políticas quanto à atualização das evidências numa forma transparente e estruturada para informar se a intervenção, recomendação clínica, decisões de coberturas de saúde, recomendações e/ou decisões de saúde pública foram projetadas de acordo com a melhor evidência disponível e alinhada com o contexto local ${ }^{(5,26)}$.

Nesta pesquisa identificou-se que cinco publicações abordaram em seus resultados os conhecimentos, habilidades e atitudes dos enfermeiros necessários para incorporar os valores e objetivos do paciente e da família na tomada de decisão clínica ${ }^{(12,13,14,16,22)}$. Destacando-se duas que exploraram a tomada de decisão compartilhada entre a equipe interdisciplinar e a pessoa com DP avançada ${ }^{(12,14)}$.

Em uma delas, foram identificados quatro temas representando as experiências atuais com o processo de tomada de decisão compartilhada na DP avançada, quais sejam: I) informações e necessidades de informação, 2) fatores que influenciam a escolha do tratamento e estratégias de decisão individuais, 3) funções de tomada de decisão e 4) barreiras e facilitadores envolvidos na tomada de decisão compartilhada ${ }^{(14)}$.

Pesquisas anteriores ${ }^{16,27,28}$ reforçam que além do fornecimento de informações sobre tratamento e condições clínicas, é papel dos enfermeiros e demais membros da equipe de cuidado da pessoa com DP envolverem ativamente estes e seus familiares no processo de cuidado, pois esta ação é fundamental para o autogerenciamento e tomada de decisão compartilhada para melhorar autoeficácia, qualidade de vida, adesão ao tratamento e satisfação do paciente.

$\mathrm{Na}$ condução desta pesquisa foi possível verificar uma revisão ${ }^{(22)}$ que utilizou um modelo de prática baseada em evidências, para responder à questão clínica do reconhecimento da ansiedade em pacientes com DP, por meio do Modelo Stetler. O Modelo Stetler forneceu estrutura para identificar pesquisas pertinentes para apoiar o uso de ferramentas de avaliação da ansiedade que poderia ser implementada na prática clínica de enfermagem, meIhorando o manejo da ansiedade na DP.

Quanto as intervenções de enfermagem que abordam estratégias de decisão individual e compartilhada com o familiar, um estudo avaliou a viabilidade de cuidados ao cônjuge da pessoa com DP antes e após a estimulação cerebral profunda para DP.As principais atividades da intervenção foram um diário para registro de dúvidas cotidianas e reuniões individualizadas entre enfermeiros, pacientes e seus cônjuges com foco na rotina diária e expectativas para a estimulação cerebral profunda ${ }^{(13)}$.

A tomada de decisão compartilhada em pessoas com doença crônica está ligada à segurança do paciente e visa incluir os pacientes nas decisões do processo saúde-doença, garantindo que obtenham os cuidados que desejam. (29) $\mathrm{Na}$ DP, principalmente na fase avançada, é fundamental combinar evidências com a experiência do profissional e as preferências do paciente. Esta abordagem, combinada com suporte à informação sobre os efeitos dos tratamentos avançados na qualidade de vida ajudará pacientes e suas famílias a integrar à escolha do tratamento. ${ }^{(30)}$

Esta revisão integrativa aborda uma lacuna na literatura ao descrever adequadamente como o conhecimento de pesquisas de enfermagem baseada em evidência pode ser transladado para enfermeiros no cuidado a pessoa com DP. No entanto, certas limitações devem ser reconhecidas, a destacar: a pesquisa de dados limitou-se a três idiomas e, portanto, alguns artigos relevantes, publicados em outros idiomas demonstrando adoção de práticas baseada em evidências por enfermeiros no cuidado a pessoa com DP podem não ter sido identificados. Associado a este fator, a estratégia de busca usada para esta revisão pode não ter resultado em uma lista exaustiva de práticas baseada em evidências por enfermeiros.

Para pesquisas futuras, recomenda-se o desenvolvimento de protocolos clínicos, diretrizes terapêuticas e ferramentas de resultados de gerenciamento de enfermagem a pessoa com DP, no contexto nacional, ou a tradução e validação para o português das já existentes em outros idiomas. 


\section{CONCLUSÃO}

Os resultados apresentados comprovam que é oportuno a criação de protocolos ou diretriz de conhecimento de melhor evidência para o cuidado de enfermagem a pessoa com DP. Apesar de não terem sido encontrados estudos nacionais, constatou-se que modelos existentes e descritos na literatura atual podem ser base para o desenvolvimento de práticas de enfermagem voltadas para a realidade brasileira, especialmente no cenário da Atenção Primária de Saúde.

A revisão conclui que a pesquisa baseada em evidências no cuidado de enfermagem as pessoas com DP constantemente produzem novos achados, achados para aprofundar as habilidades na prática clínica, e a adoção de transferência e intercambio de conhecimento para o cuidado de enfermagem a pessoa com doença de Parkinson envolve elaboração de diretrizes e protocolos de cuidado, iniciativas de educação permanente sobre a as características da doença, mútua ação com a equipe multiprofissional e engajamento do paciente e seus familiares no processo de viver com a doença.

Conflito de interesses: destaca-se que não há conflito de interesses.

Financiamento: bolsa de doutorado da Fundação de Amparo à Pesquisa e ao Desenvolvimento Científico e Tecnológico do Maranhão - FAPEMA. 


\section{REFERÊNCIAS}

I. Rogers G, Davies D, Rosa J, Cooper, P. Parkinson's disease: summary of updated NICE guidance. BMJ [Internet]. 2017[acesso em 12 jul 2019]; 27: I-7. Disponível em: https://doi.org/I0.1 I36/bmj.j1951

2. Van der Marck MA, Bloem BR, Borm GF, Overeem S, Munneke M, Guttman M. Effectiveness of multidisciplinary care for Parkinson's disease: a randomized, controlled trial. MDS Journals [Internet]. 20I3[acesso em 12 jul 20I9]; 28 (5): 605-6 I I. Disponível em: https://doi.org/10.1002/mds.25194

3. Hellqvist C, Berterö C. Support supplied by Parkinson's disease specialist nurses to Parkinson's disease patients and their spouses. Appl. Nurs. Res [Internet]. 2015[acesso em 12 jul 20I9]; 28(2):86-9।. Disponível em: https://doi.org/10.1016/j.apnr.2014.12.008

4. Lennaerts H, Groot M, Rood B, Gilissen K, Tulp H, Van Wensen E, et al. Guideline for Parkinson's Disease Nurse Specialists, with Recommendations for Clinical Practice. J Parkinsons Dis [Internet]. 2017[acesso em I 2 jul 2019]; 7 (4): 749-754. Disponível em: https://doi.org//0.3233 / JPD171195

5. Oelke ND, Lima MADS, Acosta AM. Translação do conhecimento: traduzindo pesquisa para uso na prática e na formulação de políticas. Rev. Gaúcha Enferm [Internet]. 20I5[acesso em I2 jul 2019]; 36 (3): II3-II7. Disponível em: https://seer.ufrgs.br/RevistaGauchadeEnfermagem/article/view/55036

6. Shin JY, Habermann B. Nursing Research in Parkinson's Disease From 2006 to 2015:A Systematic Review. Clin Nurs Res [Internet]. 20 I7[acesso em I2 jul 2019]; 26 (2): I42-I56. Disponível em: https://doi.org/I0.1 I77//0547738/66349/2

7. Kothari A, Wathen CN. A critical second look at integrated knowledge translation. Health Policy [Internet]. 2013; 109: |87-19|. Disponível em: https://doi.org/10.1016/j.healthpol.2012.11.004

8. Canadian institutes of health research. Guide to Knowledge Translation Planning at CIHR: Integrated and end-of-Grant Approaches [Internet]. 2015 [acesso em I 2 feb 20l9]. Disponível em: http://www.cihr-irsc.gc.ca/e/4532I.html

9. Gosnell R, Lazear J, Hemphill JC, Dotson D. Development of guidelines for improving oral health in individuals with Parkinson's disease. Gerodontology [Internet]. 2019[acesso em 12 jul 2019]; 36 (3): 229-235. Disponível em: https:// doi.org/I0.1 I I I/ger.I240 I

10. Whittemore R, Knafl K. The integrative review: updated methodology. J. adv. Nurs [Internet]. 2005[acesso em 12 feb 2019]; 52 (5): 546- 553. Disponível em: https://doi.org/I0.I I I I/j. I365-2648.2005.0362 I.x

I I. Connor KI, Cheng EM, Barry F, Siebens HC, Lee ML, Ganz DA, et al. Randomized trial of care management to improve Parkinson disease care quality. Neurology [Internet]. 2019[acesso em I2 feb 2019]; 92 (I6): I83 I-I842. Disponível em: https://doi.org//0.1212/WNL.0000000000007324
12. Fleisher J, Barbosa W, Sweeney MM, Oyler SE, Lemen AC, Fazl A, et al. Interdisciplinary Home Visits for Individuals with Advanced Parkinson's Disease and Related Disorders. J.Am. Geriatr. Soc [Internet]. 2018[acesso em I2 jul 2019]; 66 (6): I226- I232. Disponível em: https://doi.org/ I0. I I I I/jgs. I 5337

13. Haahr A, Østergaard K, Kirkevold M. "It Is Hard Work, But It Is Worth It": Patients and Spouses' Experiences of a Nursing Intervention to Promote Adjustment to Deep Brain Stimulation for Parkinson's Disease-A Feasibility Study. Adv Nurs Sci [Internet]. 20 I8[acesso em 12 jul 2019]; 4 (2): I74- I87. Disponível em: https:// doi.org//0.1097 / ANS.0000000000000208

14. Nijhuis FA, Van Heek J, Bloem BR, Post B, Faber MJ. Choosing an Advanced Therapy in Parkinson's Disease; is it an Evidence-Based Decision in Current Practice? J Parkinsons Dis [Internet]. 2016[acesso em I2 jul 2019]; 6 (3): 533-43. Disponível em: https://content.iospress.com/articles/journal-of-parkinsons-disease/jpd I60816

I5. Cohen EV, Hagestuen R, González-Ramos G, Cohen HW, Bassich C, Book E, et al. Interprofessional education increases knowledge, promotes team building, and changes practice in the care of Parkinson's disease. Parkinsonism Relat. Disord [Internet]. 2016[acesso em I2 jul 2019]; 22: 2I-27. Disponível em: https://doi.org//0.1016/j.parkreldis.2015.11.001

16.Van der Marck MA, Bloem BR. How to organize multispecialty care for patients with Parkinson's disease. Parkinsonism Relat. Disord [Internet]. 2014[acesso em 12 feb 2019]; 20: SI67-SI73. Disponível em: https://doi.org/I0.1016/SI3538020(13)70040-3

17. Van der Marck MA, Klok MPC, Okun MS, Giladi N, Munneke $M$, Bloem BR, et al. Consensus-based clinical practice recommendations for the examination and management of falls in patients with Parkinson's disease. Parkinsonism Relat. Disord [Internet]. 20 I4[acesso em 12 out 2019]; 20 (4): 360-369. Disponível em: https://www.movementdisorders. org/MDS-Files I/Education/PDFs/2nd-Annual-Allied-Health -Professionals-Summer-School-20 I4/BloemLecture3-I vanderMarck_PRD20I4fallstaskforce.pdf

18. Chenoweth L, Sheriff J, Mcanally L, Tait F. Impact of the Parkinson's disease medication protocol program on nurses' knowledge and management of Parkinson's disease medicines in acute and aged care settings. Nurse Educ Today [Internet]. 2013[acesso em I 2 out 20I9]; 33 (5): 458-464. Disponível em: https://doi.org/I0.1016/j.nedt.2012.04.022

19. Gregory P, Morgan K, Lynall A. Improving sleep management in people with Parkinson's. Br J Community Nurs [Internet]. 2012[acesso em 12 out 20I9]; 17 (I): I4-20. Disponível em: https://doi.org/10.12968/bjcn.2012.17.I.14

20. Lindop F. A multidisciplinary approach to Parkinson's disease. BJNN [Internet]. 2012[acesso em 12 out 2019]; 8 (2): 6I-64. Disponível em: https://doi.org//0.12968/ bjnn.20I2.8.2.6I 
21. Hermanns M. Culturally competent care for Parkinson disease. Nurs Clin North Am. [Internet]. 201 I [acesso em 12 out 2019]; 46 (2): I7I-I80. Disponível em: https://doi.org/l0.1016/j.cnur.201I.02.003

22. Snyder CH, Facchiano L, Brewer M. Using evidence-based practice to improve the recognition of anxiety in Parkinson's disease. J Nurse Pract [Internet]. 201 I [acesso em 12 out 2019]; 7 (2): I36-|4I. Disponível em: https://doi. org/10.1016/j.nurpra.2010.08.017

23. Viscomi P, Jeffrey J. Development of clinical practice guidelines for patient management of blood pressure instability in multiple system atrophy, Parkinson's disease, and other neurological disorders. Can. J. Neurosci. Nurs [Internet]. 2010[acesso em I2 feb 2019]; 32 (2): 6-19. Disponível em: https://www.ncbi.nlm.nih.gov/pubmed/2053364 I

24. Weiss ME, Bobay KL, Johantgen M, Shirey MR. Aligning Evidence-Based Practice with translational research: Opportunities for Clinical Practice Research. JONA [Internet]. 2018 [acesso em 12 jul 2019]; 48 (9):425-43 I. Disponível em: https://journals.Iww.com/jonajournal/Fulltext/20I8/09000/ Aligning_Evidence_Based_Practice_With.6.aspx

25. Straus S, Tetroe J, Graham ID. Knowledge translation in health care: moving from evidence to practice. John Wiley \& Sons. 2013.

26. Abreu DMFD, Santos EMD, Cardoso GCP, Artmann E. Usos e influências de uma avaliação: translação de conhecimento? Saúde em Debate [Internet]. 20I7[acesso em I2 jul 2019];4I:302316. Disponível em: https://doi.org//0.1590/0103-I I042017s22.
27. Hammerschmidt KSDA, Ferreira JM, Heidemann ITSB,Alvarez AM, Locks MOH., Siewert, J. S. Gerontotecnologia para prevenção de quedas dos idosos com Parkinson. Rev. Bras Enferm [Internet]. 2019[acesso em 21 apr 2020];72:243250. Disponível em: https://doi.org//0.1590/0034-7/672018-0704

28. Nunes SFL, Alvarez AM, Valcarenghi RV, Hammerschmidt KSDA, Baptista R. Adaptação dos Familiares Cuidadores de Idosos com Doença de Parkinson: Processo de Transição. Psic.: Teor e Pesq. [Internet]. 2019[acesso em 21 apr 2020]; 35(SPE). Disponível em: https://doi.org/10.1590/0102.3772e35nspe4

29. Ferreira RJ, Santos EJ,Wit M, Marques A, Barbieri-Figueiredo MDC, Marques A, Ndosi M. Shared decision-making in people with chronic disease: Integrating the biological, social and lived experiences is a key responsibility of nurses. Musculoskeletal care [Internet]. 2020[acesso em 21 apr 2020]; I8(I): 84. Disponível em: https://onlinelibrary.wiley. com/doi/full/I0.1002/msc. 1443

30. Nijhuis FA, van den Heuvel L, Bloem BR, Post B, Meinders MJ. The patient's perspective on shared decision-making in advanced Parkinson's disease: a cross-sectional survey study. Front neurol [Internet]. 2019 [acesso em 21 apr 2020];10: 896. Disponível em: https://www.ncbi.nlm.nih. gov/pmc/articles/PMC6706819/

Recebido: 2020-03-23

Aceito: 2020-04-22 